\title{
Clinical, pathological and genetic characteristics of a pedigree with myotonic dystrophy type 1
}

\author{
QING LIU, YU-FEI ZHENG, YAN-PING ZHU, SHI-QING LING and WEI-RONG LI \\ Department of Neurology, Taiyuan Central Hospital of Shanxi Medical University, Taiyuan, Shanxi 030009, P.R. China
}

Received October 23, 2014; Accepted July 23, 2015

DOI: $10.3892 /$ etm.2015.2738

\begin{abstract}
The aim of the present study was to investigate the clinical, pathological and molecular genetic characteristics of a pedigree with myotonic dystrophy type 1 (DM1). A series of clinical data from a pedigree with DM1 were collected. Muscle biopsy revealed a typical nuclear ingression within numerous muscle fibers following hematoxylin and eosin staining. Genomic DNA was extracted from the venous blood of two patients and the triplet-primed polymerase chain reaction method was performed to amplify the dystrophia myotonic protein kinase (DMPK) gene. The amplified products were subjected to gene sequencing by capillary fluorescence electrophoresis, and a pathogenic mutation in the DMPK gene comprising >50 cytosine-thymine-guanine repeat sequences was found. DM1 includes multi-system damage, as well as skeletal muscle involvement, and can affect the central nervous system, endocrine glands, skin and heart. A skeletal muscle biopsy and genetic testing can confirm the diagnosis and clarify the severity of the disease. In addition, it is necessary to distinguish DM1 from DM2.
\end{abstract}

\section{Introduction}

Myotonic dystrophy (DM) is an autosomal dominant genetic disease that involves multiple systems, and is the most common type of adult muscular dystrophy. DM can be divided into DM type 1 (DM1) and DM type 2 (DM2) on the basis of clinical features and genetic differences. DM1 was first described by Steinert in 1909; thus it is also known as Steinert's disease (1). Its causative gene is the dystrophia myotonic protein kinase (DMPK) gene, located on chromosome 19. The disease is caused by an increased number of repeat sequence copies of trinucleotides [cytosine-thymine-guanine, $(\mathrm{CTG})_{\mathrm{n}}$ ] in the 3'-untranslated region of the DMPK gene (2). Healthy

Correspondence to: Dr Wei-Rong Li, Department of Neurology, Taiyuan Central Hospital of Shanxi Medical University, 1 Lane Three, Taiyuan, Shanxi 030009, P.R. China

E-mail: weironglicn@126.com

Key words: myotonic dystrophy type 1, myotonia, skeletal muscle pathology, nuclear ingression, myotonic dystrophy type 2 individuals have 5-37 CTG repeat sequences, and $>37$ is considered an abnormal number of CTG repeat sequences. Individuals with 38-49 repeat sequences show no clinical symptoms, however their offspring may have a substantial risk of exhibiting the clinical symptoms of DM. Patients with $>50$ repeat sequences would show clinical symptoms (3).

In 1994, Ricker et al (4) found that in certain DM1-like patients proximal myotonia was observed, and the numbers of $(\mathrm{CTG})_{\mathrm{n}}$ repeat sequence copies in the untranslated regions of DMPK were in the normal range; thus the term 'proximal myotonic myopathy' was applied to this condition (5). In 2001, Liquori et al (6) found that the affected gene in such patients was located on intron 1 of the chromosome 3 finger protein 9 gene, and the disease has been observed to develop as the number of copies of the tetranucleotide CCTG repeat sequence increases (7); thus, the condition was renamed as DM2.

The molecular mechanisms of DM are complex. For both DM1 and DM2, the repeatedly amplified sequences are located in the untranslated regions of disease-causing genes, and do not change the protein encoding of their location genes. The mechanism by which the amplification of the repeat sequence leads to the increased incidence of DM is debatable. Among known patients with DM1, three mechanisms may lead to clinical symptoms (8): i) DMPK protein haplotype insufficiency. The increased repeat sequence amplification may affect the gene transcription of DMPK or retain the DMPK gene transcripts within the nucleus without transporting them into the cytoplasm, thus reducing the expression of DMPK protein, causing haplotype insufficiency, and leading to clinical symptoms. ii) The abnormally repeated sequence amplification could affect the structures of chromosomes, thus affecting the expression of DMPK genes and genes in the adjacent region. For example, the expression of SIX5 gene, adjacent to the DMPK gene area in patients with DM1, is downregulated. SIX5 is also similar to the eye-development gene of Drosophila and mouse gene families that regulate distal limb muscle development. Since cataract and distal end malnutrition are common symptoms of DM1, it has been hypothesized that SIX5 haplotype insufficiency induces DM1 pathogenesis. SIX5-knockout mice, however, only exhibit cataract symptoms, without multi-system damage, so this mechanism is not sufficient to cause the characteristic multi-system damage of DM1. iii) RNA toxicity and abnormal shearing. The abnormal repeatedly amplified sequence may be transcribed into mRNA that contains an amplified 
CUG repeated sequence. This mRNA would be abnormally accumulated in the nucleus, thus changing the activities of certain RNA-binding proteins, for example, increasing the numbers of CUG-binding protein/Elav-like family member 1 , and decreasing the expression of muscle-blind-like protein (MBNL1). MBNL1 inside the cytoplasm would then transfer to the nucleus to compensate for the reduction of MBNL1. The reduction of MBNL1, as well as the synergistic effects increased by the CUG binding protein, would interfere with the normal cutting process, resulting in error splicing of sub-transcripts and causing multi-system damage. For example, it could cause the abnormal expression of: Chloride channels, leading to myotonia; troponin, generating myocardial damage; or insulin receptors, resulting in insulin resistance (8). In addition to these three mechanisms, various other mechanisms have also been proposed to be involved in the pathogenesis of DM1, such as transcription factor dysfunction, microRNA dysfunction, RNA interference and double-stranded RNA mechanisms, cell stress and the inhibition of translation. The synergistic effects of various mechanisms are likely to induce the multi-system damage associated with DM1.

The main symptoms of DM are muscle weakness, muscle atrophy, myotonia and multi-system effects. Following repeated muscle contractions, the myotonia phenomenon can be improved, known as the 'warm-up phenomenon' (9). The main manifestations of multi-system effects are alopecia, cataracts, sexual dysfunction, heart block, mental retardation, hyperthyroidism and digestive symptoms. Electrophysiological examination by needle electromyography (EMG) demonstrates myotonia potential and myogenic damage. The nerve conduction velocity is generally normal. Pathological examination of muscles indicates variations in muscle fiber diameter, showing a bimodal distribution. Hypertrophy and atrophy may be observed. Nuclear ingression is observed in some muscle fibers, and in certain cases, cytoplasmic bodies in the muscle fibers, nuclear chain formation, circular muscle fibers, selective type I muscle fiber atrophy, non-merging necrosis and regeneration may be observed (10). Magnetic resonance imaging examination of the muscles has shown that the affected muscles exhibit fatty degeneration (11).

In general, southern blot analysis is used to detect the amplified gene sequences (12). This method has high specificity, and is able to estimate the number of repeat units of CTG, although the sensitivity is low and the method is prone to producing false-negative results. Additionally, it is time-consuming, and requires the application of radioactive probes. Furthermore, the triplet-primed polymerase chain reaction (TP-PCR) method has been used, which is simple and rapid (13), although it cannot provide accurate numbers of CTG repeat sequences. In order to eliminate false-negative results, southern blot analysis could be performed on patients with negative TP-PCR results to ensure the reliability of results.

The present study summarizes the clinical symptoms, skeletal muscle pathology and genetic testing information of a pedigree with DM1, treated in the Department of Neurology, Taiyuan Central Hospital (Taiyuan, China) between March 2014 and April 2014, with the aim of further exploring the pathogenesis. The clinical, pathological and genetic testing features of DM1, as well as how to distinguish it from DM2 and other myopathies involving myotonia, are discussed, to further deepen the understanding and awareness of this type of disease.

\section{Materials and methods}

Clinical data. The clinical data of a pedigree with DM1, treated in the Department of Neurology, Taiyuan Central Hospital of Shanxi Medical University (Taiyuan, China) from March 2014 to April 2014, were collected. The present study was conducted in accordance with the Declaration of Helsinki and with approval from the Ethics Committee of Shanxi Medical University. Written informed consent was obtained from all participants.

Muscle pathology. The proband underwent an open skeletal muscle biopsy of the left bicep brachii under local anesthesia. The sample was then rapidly frozen in liquid nitrogenisopentane, and sliced into $7-\mu \mathrm{m}$ frozen serial sections. Hematoxylin and eosin staining was performed prior to pathological analysis under a Nikon Eclipse Ni-U light microscope (Nikon Corporation, Tokyo, Japan) (10). The pathological features of DM1 patients were analyzed.

\section{Genetic testing}

DNA extraction. Venous blood for DNA extraction was sampled from the proband and his sister, and was subjected to TP-PCR analysis to amplify the DMPK gene (12). A whole blood genomic DNA extraction kit (Shanghai Shenggong, Shanghai, China) was used to extract genomic DNA for the PCR amplification. The DNA was dissolved and stored in $10 \mathrm{mmol} / \mathrm{l}$ Tris- $\mathrm{HCl}$ solution $(\mathrm{pH} \mathrm{7.5)}$. The sequences of primers (Shanghai Shenggong) were: P1, 5'-GGGGCTCGA AGGGTCCTTGT-3'; P2, 5'-GTGCGTGGAGGATGGAAC ACG-3'; P3R, 5'-AGCGGATAACAATTTCACACAGGA-3'; P4CAG, 5-AGCGGATAACAATTTCACACAGGACAGCAG CAGCAGCAGCAG-3'. The 5' terminus of P1 was labeled with carboxyfluorescein (14).

$P C R$ reaction. The $25-\mu 1$ reaction system contained 0.5 units KAPA HiFi HotStart DNA Polymerase (KAPA Biosystems, Boston, MA, USA), $600 \mu \mathrm{mol} / 1$ deoxynucleotide triphosphates and 5.0 $\mu 1$ 5X KAPA GC buffer (containing 1X $2.0 \mathrm{mmol} / \mathrm{l} \mathrm{Mg}^{2+}$; KAPA Biosystems). The concentration of template DNA was 50-500 ng/cycle. Within the PCR-P1P2 reaction system, the primers included $\mathrm{P} 1$ and $\mathrm{P} 2$, and their final concentration was $0.6 \mu \mathrm{mol} / 1$ for both. Within the TP-PCR reaction system, the primers included $\mathrm{P} 1, \mathrm{P} 3 \mathrm{R}$ and $\mathrm{P} 4 \mathrm{CAG}$, with the final concentrations of $0.8,0.6$ and $0.2 \mu \mathrm{mol} / 1$, respectively. The sample was pre-degenerated at $95^{\circ} \mathrm{C}$ for $5 \mathrm{~min}$, prior to entering the PCR cycle. The parameters of the cycle were: $98^{\circ} \mathrm{C}$ for $20 \mathrm{sec}, 65^{\circ} \mathrm{C}$ for $30 \mathrm{sec}$ and $72^{\circ} \mathrm{C}$ for $2 \mathrm{~min}$, for a total of 30 cycles; followed by an extension at $72^{\circ} \mathrm{C}$ for $10 \mathrm{~min}$. The products were stored at $4^{\circ} \mathrm{C}$.

Genetic analysis. The amplification products were analyzed by capillary electrophoresis in an ABI 3730 sequencer (Applied Biosystems Life Technologies, Foster City, CA, USA). One microliter of PCR products was mixed with $9 \mu 1$ formamide, and capillary electrophoresis was performed following 4 min degeneration. GeneScan 500-LIZ dye Size Standard (Life Technologies) was used for the reproducible sizing of fragment analysis data, and ABI GeneMapperID 
version 3.2 (Applied Biosystems) was used to conduct the analysis.

\section{Results}

\section{Patient III 1}

The proband was a 38-year-old male, and is shown in the pedigree chart as III 1 (Fig. 1).

Clinical manifestations. The patient had experienced upper limb weakness for 20 years, mostly in the distal upper limbs. Following hard clenching, the patient was unable to loosen his fists. This manifestation progressively increased, and the patient gradually exhibited atrophy in the proximal upper and lower limbs, and shoulder and back muscles. Lower extremity weakness appeared 6 years ago.

Medical history. For the past 20 years, the left side of the face of the patient exhibited peripheral paralysis. In addition, hearing was lost in the right ear, and baldness gradually developed.

Personal history. The patient was married, without children, and sexual dysfunction was observed following the marriage.

Family history. The grandfather of the patient had symptoms of upper limb weakness, and succumbed to stomach cancer. The mother of the patient had symptoms of upper limb weakness, and succumbed to pulmonary heart disease. The patient had a sister who exhibited similar symptoms.

Admission examination. The patient had left peripheral facial paralysis, without a typical thin and angular face. The neck flexor force was grade 2 . The muscle strength of the proximal ends of the upper limbs was grade 4-, the finger dorsal extensor muscle strength was grade $4+$, the muscle strength of the proximal end of the lower extremity was grade 5, the dorsal extensor strength of the foot was grade 2 and the plantar flexor force was grade 4 . The bilateral supraspinatus, infraspinatus muscle, deltoid, biceps brachii and triceps brachii exhibited atrophy, and the bilateral gastrocnemius showed slight hypertrophy. The fingers exhibited the myotonic phenomenon (Figs. 2 and 3), while the knotted muscles were not fully released. The tendon reflex of the bilateral upper limbs was not elicited, and the knee tendon reflex was reduced.

Laboratory information. Regarding the thyroid functions, the thyroid-stimulating hormone level was $5.36 \mu \mathrm{IU} / \mathrm{ml}$, while the thyroxine level exhibited no evident abnormality. Biochemical examination showed that the alanine aminotransferase activity was $62 \mathrm{U} / 1$, aspartate aminotransferase activity was $56.6 \mathrm{U} / 1$ and uric acid level was $421 \mu \mathrm{mol} / 1$. In addition, the triglyceride level was $8.65 \mathrm{mmol} / 1$, total cholesterol level was $5.95 \mathrm{mmol} / \mathrm{l}$, fasting blood glucose was $5.7 \mathrm{mmol} / 1$, creatine kinase activity was $180 \mathrm{U} / 1$, lactate dehydrogenase activity was $246 \mathrm{U} / \mathrm{l}$ and the erythrocyte sedimentation rate was $20 \mathrm{~mm} / \mathrm{h}$. The limb EMG showed myotonia, which was suspected to be myogenic impairment. Consultation with the Department of Ophthalmology of Taiyuan Central Hospital of Shanxi Medical University resulted in a diagnosis of 'bilateral cataracts (congenital)'.

Muscle pathology. Under the light microscope, it was observed that the muscle fibers varied in size and diameter, with a bimodal distribution. Some of the muscle fibers were hypertrophic and others were rounded and exhibited atrophy.

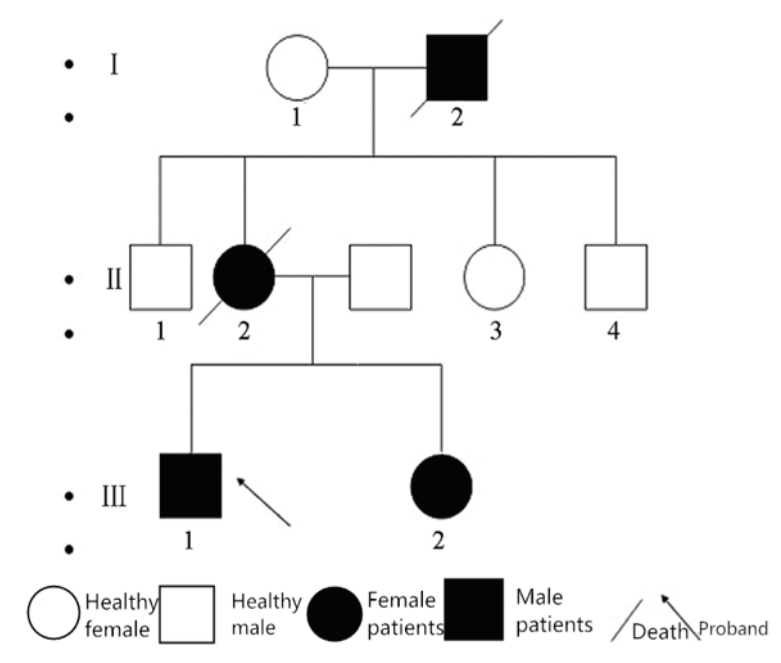

Figure 1. Pedigree chart of myotonic dystrophy.

A large number of muscle fibers had undergone nuclear ingression. Nuclear aggregation was occasionally visible, but necrosis, regeneration and whorled muscle fibers were not evident. In addition, there were no cytoplasmic bodies inside the muscle fibers (Fig. 4).

Patient III 2. The sister of the proband, shown as III 2 in Fig. 1, was 30 years old and had experienced mild weakness of the upper limbs since the age of 24 years. The patient was not able to release her fists following hard clenching. A physical examination showed that the muscle strength of the distal parts of the upper limbs was grade 5-, while that of the proximal part was grade 5. The muscle strength of the lower extremities was grade 5 .

Patient II 1. The mother of the proband, shown as II 1 in Fig. 1, experienced disease onset at the age of 40 years, and succumbed to pulmonary heart disease at the age of 65 years. The clinical manifestations in this patient were upper limb weakness, and the inability to release the fists following hard clenching.

Patient I 2. The grandfather of the proband, shown as I 2 in Fig. 1, experienced disease onset at the age of 45 years, and succumbed to stomach cancer at 72 years. The clinical manifestations were upper limb weakness, and the fists could not be released following hard clenching.

Genetic test results. Genetic testing of the proband and his sister found that the DMPK gene exhibited the disease-causing mutation $\mathrm{CTG}$ with $>50$ repeat units.

\section{Discussion}

DM is an autosomal dominant genetic disease that normally involves multiple systems. It can be divided into DM1 and DM2 according to its clinical features and genetic profiles.

According to the clinical manifestations, DM1 can be further divided into four types: Asymptomatic, congenital, child-onset and adult-onset (15). Asymptomatic DM1 patients have no clinical symptoms. Patients with congenital DM1 may 


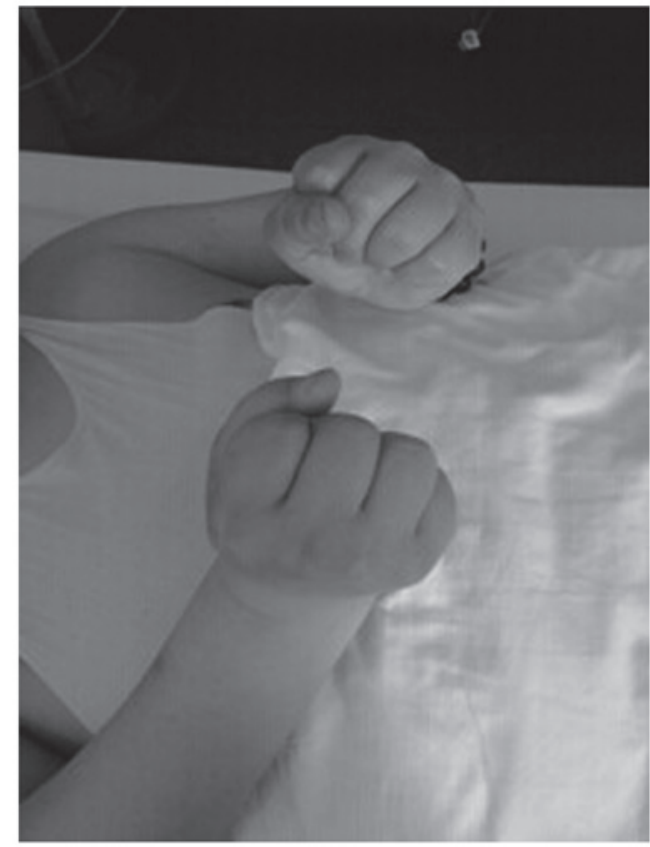

Figure 2. Clasped hands of the proband.

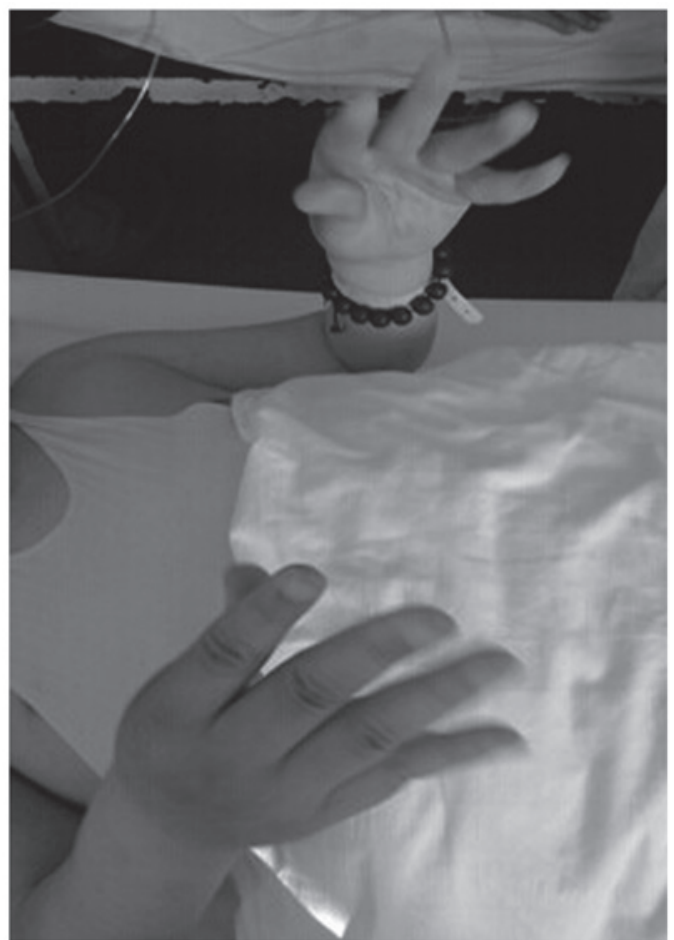

Figure 3. Unclasping of the hands of the proband was delayed, exhibiting the myotonic phenomenon.

exhibit excessive amniotic fluid or fetal reduction prior to birth and, after birth, they may show severe limb weakness, low muscle tone and respiratory failure. After birth, the patients may experience muscular hypotonia of the limbs and trunk and respiratory, facial and bulbar muscles. Due to facial weakness, the patients may exhibit a V-shaped upper lip (also known as a tent- or fish-shaped upper lip). Due to weakness in the chewing muscles, there may be a difficulty in sucking, resulting in feeding problems. The respiratory muscle weakness may

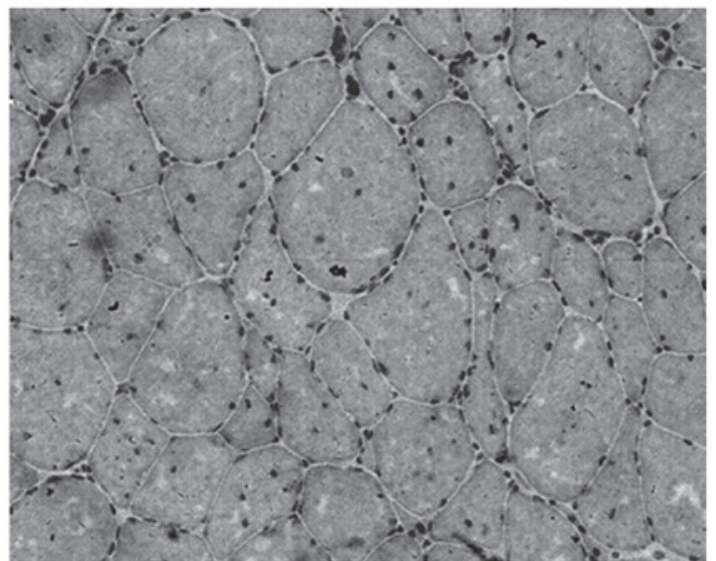

Figure 4. Skeletal muscle biopsy of the left biceps brachii of the proband The muscle fibers varied in size and diameter, and exhibited a bimodal distribution. Some muscle fibers exhibited hypertrophy, while other muscle fibers exhibited were small, rounded and atrophic. A large number of muscle fibers had undergone nuclear ingression. Nuclear aggregation was occasionally visible, while necrosis, regeneration and vortex muscle fibers were not observed. In addition, there were no cytoplasmic bodies inside the muscle fibers. Hematoxylin and eoxin staining; magnification, x100.

cause the patients to exhibit respiratory failure neonatally. The typical myotonic phenomenon may not necessarily appear in the early stages. The majority of patients succumb at an early stage; surviving patients may suffer from mental retardation, and exhibit cerebral atrophy and ventricular contraction (16), followed by severe respiratory and circulatory complications in their 30s and 40s.

Patients with child-onset DM1 exhibit onset in their childhood, when the symptoms of muscle weakness, myotonia and muscle atrophy are not evident, so the condition can be challenging to diagnose. Facial muscle involvement is the most common symptom, although the typical V-shaped upper lip of congenital DM1 is not observed. Dysarthria and hand myotonia are also typical manifestations, and are often accompanied by a movement development delay and mental retardation (17).

The adult-onset type generally exhibits the symptoms of typical multi-system damage, which could involve the skeletal muscle, heart, endocrine glands, skin, central nervous system, eyes and digestive system. Skeletal muscle involvement may appear as weakness in the distal parts of all four limbs, leading to non-flexible fine motions of the upper extremities, and foot drop. Furthermore, the levator muscle of the upper eyelid may be involved, causing ptosis of the upper eyelid. The masticatory muscle may be involved leading to atrophy of the temporalis and masticatory muscles, elevated cheekbones, and thinning of the face (the typical 'hatchet face'). Atrophy of the sternocleidomastoid muscle may cause a slender neck, associated with a reduction in craning muscle strength and excessive forward protrusion, thus forming a 'goose-neck'. Extraocular muscle involvement is relatively rare, and normally not accompanied by gastrocnemius muscle hypertrophy (12). The muscle weakness slowly progresses, and a small number of patients may combine the symptoms of four-limb myasthenia, which is aggravated by neuraxial peripheral neuropathy (18). Certain patients may exhibit symptoms of myotonia. The myotonic phenomenon refers to a delayed relaxation of the skeletal muscle following voluntary contraction. The myotonic 
phenomenon of patients with DM1 normally appears in the distal ends of limbs, and mostly happens when the fingers are clenched, for example when using tools or rotating a doorknob. When the muscles are repeatedly contracted, myotonia can be improved, known as the 'warm-up phenomenon' (9). The sustained contraction of muscle due to compression with a percussion hammer, where the muscles contract and then relax a few seconds later, is known as percussion myotonia. The eyelid muscles may also suffer myotonia, as shown when the eyes cannot be opened immediately when closed with force.

The proband in the present study exhibited typical myotonia, including the warm-up phenomenon, muscle weakness mainly in the distal ends of limbs, and muscle atrophy mostly in the proximal ends of limbs, which were all in line with the typical skeletal muscle performance of DM1. However, the proband also exhibited mild hypertrophy in the bilateral gastrocnemius, which is not a usual symptom of patients with DM1. Thus, further accumulation of data is required to identify whether patients with DM1 may exhibit hypertrophy of the bilateral gastrocnemius.

In addition to skeletal muscle involvement, patients with DM1 may exhibit symptoms of multi-system involvement. Congenital cataracts may appear, and patients may exhibit cardiac symptoms, such as conduction block or tachyarrhythmia, sudden cardiac mortality, myocardial disease or ischemic heart disease (19). Cardiac pathological examination may reveal cardiac fibrosis, cardiac hypertrophy and the accumulation of fatty tissue in the conducting system and sinoatrial node (20). Electrocardiography may show a prolonged PR interval, widened QRS wave, paroxysmal or persistent atrial fibrillation and various malignant arrhythmias, which may often lead to sudden cardiac mortality. Patients with DM1 may also experience endocrine abnormalities, affecting the thyroid, pancreas, hypothalamus and gonads. Thyroid involvement may cause thyroid dysfunction; pancreatic involvement may cause insulin resistance resulting in abnormal glucose tolerance; and gonad involvement may cause testicular atrophy in male patients, leading to sexual dysfunction and even infertility, and female patients may suffer from spontaneous abortion and menstrual abnormalities. The involvement of the skin may result in alopecia, which is much more common in male patients. Central nervous system involvement may commonly be exhibited as mental retardation, daytime sleepiness and night-time sleep disorders. The patients may also have personality disorders, manifested as compulsion, irritability or apathy (21). Digestive system involvement may include cholecystitis, cholelithiasis, abnormal liver functions, intestinal pseudo-obstruction and congenital megacolon, and also delayed gastric emptying, diarrhea and other symptoms.

In the present study, the proband exhibited an elevated level of thyroid-stimulating hormone, while the thyroxine level was normal, suggesting the existence of subclinical hypothyroidism. The increase in alanine aminotransferase and aspartate aminotransferase prompted abnormal liver function, associated with congenital cataracts, baldness and sexual dysfunction, which was consistent with the multi-system involvement of DM1. In patients with DM1, serological examination typically shows a mild to moderate elevation of creatine kinase, an increase in the activities of aspartate aminotransferase and alanine aminotransferase, and a reduction in the levels of male serum testosterone and thyroid hormone (11). The proband exhibited peripheral facial paralysis and right-ear conductive deafness, however, this has not been associated with DM1 previously; thus, whether a correlation with DM1 exists requires further study.

In the present study, the proband exhibited mild elevations of creatine kinase, aspartate aminotransferase, alanine aminotransferase and thyroid-stimulating hormone. EMG showed myotonic discharge, and reduction of re-contraction movement potential, with pure to mixed phases. Muscle pathology examination revealed variations in the diameters of muscle fibers, showing a bimodal distribution. Some hypertrophy of the muscle fibers was also observed. Numerous muscle fibers demonstrated nuclear ingression, and nuclear aggregation was occasionally present. However, there was no necrosis, regeneration or whorled muscle fibers, and no cytoplasmic bodies were visible inside the muscle fibers, consistent with the results of the auxiliary DM1 examinations. The TP-PCR method was able to reveal the disease-causing mutations in the proband and his sister, with $>50$ CTG repeats. Thus diagnostic confirmation was obtained and the results were sensitive and reliable. However, it is not possible to accurately determine the numbers of CTG repeat sequences using this method. In order to accurately assess the prognosis of patients and the possible severity of disease in future generations, it may be necessary to perform southern blot analysis to accurately determine the number of CTG repeat sequences.

\section{Acknowledgements}

This study was supported by the Natural Science Foundation of Shanxi Province (grant no. 2013011052-5).

\section{References}

1. Steinberg $\mathrm{H}$ and Wagner A: Hans Steinert: 100 years of myotonic dystrophy. Nervenarzt 79: 961-962, 2008 (In German).

2. Savić Pavićević D, Miladinović J, Brkušanin M, Sviković S, Djurica S, Brajušković G and Romac S: Molecular genetics and genetic testing in myotonic dystrophy type 1 . Biomed Res Int 2013: 391821, 2013.

3. Valaperta R, Sansone V, Lombardi F, Verdelli C, Colombo A, Valisi M, Brigonzi E, Costa E and Meola G: Identification and characterization of DM1 patients by a new diagnostic certified assay: Neuromuscular and cardiac assessments. Biomed Res Int 2013: 958510, 2013.

4. Ricker K, Koch MC, Lehmann-Horn F, Pongratz D, Otto M, Heine R and Moxley RT 3rd: Proximal myotonic myopathy: A new dominant disorder with myotonia, muscle weakness, and cataracts. Neurology 44: 1448-1452, 1994.

5. Kamsteeg EJ, Kress W, Catalli C, Hertz JM, Witsch-Baumgartner M, Buckley MF, van Engelen BG, Schwartz $\mathrm{M}$ and Scheffer H: Best practice guidelines and recommendations on the molecular diagnosis of myotonic dystrophy types 1 and 2. Eur J Hum Genet 20: 1203-1208, 2012.

6. Liquori CL, Ricker K, Moseley ML, Jacobsen JF, Kress W, Naylor SL, Day JW and Ranum LP: Myotonic dystrophy type 2 caused by a CCTG expansion in intron 1 of ZNF9. Science 293: 864-867, 2001

7. Kurosaki T, Ueda S, Ishida T, Abe K, Ohno K and Matsuura T: The unstable CCTG repeat responsible for myotonic dystrophy type 2 originates from an AluSx element insertion into an early primate genome. PLoS One 7: e38379, 2012.

8. Machuca-Tzili L, Brook D and Hilton-Jones D: Clinical and molecular aspects of the myotonic dystrophies: A review. Muscle Nerve 32: 1-18, 2005. 
9. Logigian EL, Blood CL, Dilek N, Martens WB, Moxley RT IV Wiegner AW, Thornton CA and Moxley RT III: Quantitative analysis of the 'warm-up' phenomenon in myotonic dystrophy type 1. Muscle Nerve 32: 35-42, 2005.

10. Meola G: Clinical aspects, molecular pathomechanisms and management of myotonic dystrophies. Acta Myol 32: 154-165, 2013

11. Udd B, Meola G, Krahe R, Wansink DG, Bassez G, Kress W, Schoser B and Moxley R: Myotonic dystrophy type 2 (DM2) and related disorders report of the 180th ENMC workshop including guidelines on diagnostics and management 3-5 December 2010, Naarden, The Netherlands. Neuromuscul Disord 21: 443-450, 2011.

12. Li M, Wang Z, Cui F, Yang F, Chen Z, Ling L, Pu C and Huang X: Investigation of molecular diagnosis in Chinese patients with myotonic dystrophy type 1. Chin Med J (Engl) 127: 1084-1088, 2014.

13. Addis M, Serrenti M, Meloni C, Cau M and Melis MA: Triplet-primed PCR is more sensitive than southern blotting-long PCR for the diagnosis of myotonic dystrophy type 1 . Genet Test Mol Biomarkers 16: 1428-1431, 2012.

14. Singh S, Zhang A, Dlouhy S and Bai S: Detection of large expansions in myotonic dystrophy type 1 using triplet primed PCR. Front Genet 5: 94, 2014.
15. Turner $\mathrm{C}$ and Hilton-Jones D: The myotonic dystrophies: Diagnosis and management. J Neurol Neurosurg Psychiatry 81: 358-367, 2010.

16. Ashizawa T: Myotonic dystrophy as a brain disorder. Arch Neurol 55: 291-293, 1998

17. Steyaert J, Umans S, Willekens D, Legius E, Pijkels E, de Die-Smulders C, Van den Berghe H and Fryns JP: A study of the cognitive and psychological profile in 16 children with congenital or juvenile myotonic dystrophy. Clin Genet 52: 135-141, 1997.

18. Krishnan AV and Kiernan MC: Axonal function and activity-dependent excitability changes in myotonic dystrophy. Muscle Nerve 33: 627-636, 2006.

19. Lund M, Diaz LJ, Ranthe MF, Petri H, Duno M, Juncker I, Eiberg H, Vissing J, Bundgaard H, Wohlfahrt J and Melbye M: Cardiac involvement in myotonic dystrophy: A nationwide cohort study. Eur Heart J 35: 2158-2164, 2014.

20. Phillips MF and Harper PS: Cardiac disease in myotonic dystrophy. Cardiovasc Res 33: 13-22, 1997.

21. Douniol M, Jacquette A, Cohen D, Bodeau N, Rachidi L, Angeard N, Cuisset JM, Vallée L, Eymard B, Plaza M, et al: Psychiatric and cognitive phenotype of childhood myotonic dystrophy type 1. Dev Med Child Neurol 54: 905-911, 2012. 